\title{
AVALIAÇÃO DE METODOLOGIAS PARA TESTES DE SELETIVIDADE DE INSETICIDAS REGULADORES DE CRESCIMENTO A TRICHOGRAMMA PRETIOSUM (RILEY, 1879) (HYMENOPTERA: TRICHOGRAMMATIDAE) NA CULTURA DA MACIEIRA EM CONDIÇÕES DE LABORATÓRIO
}

\author{
EVALUATION OF METHODOLOGIES FOR TESTINGS THE SELECTION \\ OF PRODUCTS THAT REGULATE THE GROWTH OF TRICHOGRAMMA \\ PRETIOSUM (RILEY, 1879) (HYMENOPTERA: TRICHOGRAMMATIDAE) IN THE \\ CULTIVATION OF APPLE IN LABORATORY CONDITIONS
}

\author{
Wilson José Morandi Filho' ${ }^{1}$ Cristiane Müller ${ }^{2}$; Crislaine Alves Barcellos de Lima \\ Wagner Roza Härter ${ }^{2}$; Fabrizio Pinheiro Giolo ${ }^{4}$; Anderson Dionei Grützmacher ${ }^{5}$
}

\begin{abstract}
RESUMO
Neste trabalho, foram estudados alternativas de metodologias, para verificar o efeito de inseticidas reguladores de crescimento na cultura da macieira, sobre Trichogramma pretiosum, em comparação com a metodologia padrão utilizada pela International Organization for Biological and Integrated Control (IOBC). Os inseticidas estudados foram: lufenuron/Match ${ }^{\circledR} \mathrm{CE}$ (300), methoxifenozide/Intrepid ${ }^{\circledR} 240$ SC (180), tebufenozide/Mimic 240 SC (90), flufenoxuron/Cascade 100 (100), e como inseticida padrão utilizou-se o inseticida fosforado fenthion (Lebaycid ${ }^{\circledR} 500$ ) (100). Foram testadas, duas metodologias na primeira, ovos de Anagasta kuehniella foram pulverizados com soluções inseticidas e fornecidos a fêmeas de T. pretiosum e na segunda foi fornecidos mel adicionado a inseticida a fêmeas de T. pretiosum.

Os parâmetros avaliados foram: longevidade de fêmeas, ovos parasitados, razão sexual e taxa de emergência. O inseticida Lebaycid $^{\circledR}$ foi o que apresentou maior toxicidade a T. pretiosum seguido do Match ${ }^{\circledR}$, em todas as metodologias testadas. Ambas as metodologias sugeridas neste trabalho podem ser utilizadas como alternativa à metodologia padrão da IOBC.

Palavras-chaves: pragas da macieira, parasitóides de ovos, alternativas metodológicas.
\end{abstract}

\section{ABSTRACT}

In this work there were studied alternatives of methodologies to verify the effect of insecticides growth regulators in the cultivation of the apple on Trichogramma pretiosum, in comparison with the methodology pattern used by International Organization for Biological and Integrated Control (IOBC). The studied insecticides were lufenuron/Match ${ }^{\circledR}$ CE (300), methoxifenozide/Intrepid® 240 SC (180), Tebufenozide/Mimic 240 SC (90), flufenoxuron/Cascade 100 (100), and as standard insecticide the insecticide fenthion was used (Lebaycid® 500) (100). Two methodologies were tested in the first eggs of Anagasta kuehniella they were powdered with

1 Faculdade de Agronomia "Eliseu Maciel" (FAEM), Universidade Federal de Pelotas (UFPel) Embrapa Uva e Vinho. Rua Livramento 515, CP. 130 Bento Gonçalves, RS. E-mail: wilsonmorandi@ yahoo.com.br Autor para correspondência

2 Faculdade de Agronomia "Eliseu Maciel" (FAEM), Universidade Federal de Pelotas (UFPel), Campus Universitário s/n. C. Postal 354, CEP: 96010-900, Pelotas, RS. E-mail: crismiler@yahoo.com.br e wagnerharter@gmail.com.

3 Faculdade de Agronomia "Eliseu Maciel" (FAEM), Universidade Federal de Pelotas (UFPel), Campus Universitário s/n. C. Postal 354, CEP: 96010-900, Pelotas, RS. E-mail: clima@ufpel.tche.br.

4 Faculdade de Agronomia "Eliseu Maciel" (FAEM), Universidade Federal de Pelotas (UFPel), Campus Universitário s/n. C. Postal 354, CEP: 96010-900, Pelotas, RS. E-mail: fgiolo.faem@ufpel.tche.br

5 Departamento de Fitossanidade, Faculdade de Agronomia "Eliseu Maciel” (FAEM), Universidade Federal de Pelotas (UFPel), Campus Universitário s/n. C. Postal 354, CEP: 96010-900, Pelotas, RS. E-mail: adgrutzm@ufpel.tche.br.

Fecha de Recepción: 22 Agosto 2007

Fecha de Aceptación: 14 Diciembre 2007 
insecticide solutions and supplied to females of T. pretiosum and in second step they were supplied honey added to insecticide to females of T. pretiosum. The appraised parameters were longevity of females, sponged eggs, sexual reason and emergency rate. The insecticide Lebaycid $₫$ was the one that presented larger toxicity to T. pretiosum followed by Match ${ }^{\circledR}$ in all the tested methodologies. Both methodologies suggested in this work they can be used as alternative of the standard methodology of IOBC.

Key words: apples plagues, eggs parasitoid, methodological alternatives.

\section{INTRODUÇÃO}

No sul do Brasil, a produtividade da macieira, Malus domestica (Burkh.), pode ser comprometida pela presença de artrópodes que se alimentam e/ ou se reproduzem na planta. Entre esses, merecem destaque a mosca-das-frutas Anastrepha fraterculus (Wiedemann, 1830) (Diptera: Tephritidae) e a mariposa-oriental Grapholita molesta (Busck, 1916) (Lepidoptera: Tortricidae) que se alimentam dos frutos e são consideradas pragas-chave da cultura (Ribeiro \& Flores, 2002). Além dessas espécies, outros insetos constituem pragas da macieira como a lagarta-enroladeira, Bonagota cranaodes (Meyrick, 1937) (Lepidoptera: Tortricidae), o piolho-de-são-josé, Quadraspidiotus perniciosus (Comstock, 1881) (Hemiptera: Diaspididae), o pulgão-lanígero, Eriosoma lanigerum (Hausmann, 1802) (Hemiptera: Aphididae) e o ácaro-vermelhoeuropeu, Panonychus ulmi (Koch, 1836) (Acari: Tetranychidae) (Ribeiro, 1999; Ribeiro \& Flores, 2002; Kovaleski \& Ribeiro, 2003).

O controle das pragas das frutíferas de clima temperado, incluindo a macieira, tem sido realizado com inseticidas de amplo espectro, principalmente fosforados, que apresentam sérias restrições de uso, com destaques para a elevada toxicidade, a possibilidade de deixar resíduos nos frutos, além de afetar os insetos benéficos presentes no agroecossistema (Kovaleski \& Ribeiro, 2003).

A preocupação com o uso de produtos fitossanitários seletivos e específicos é crescente, principalmente na Produção Integrada da Maçã (PIM), que preconiza condições favoráveis ao estabelecimento de inimigos naturais. Dentre estes, Trichogramma pretiosum (Riley, 1879) (Hymenoptera: Trichogrammatidae) destaca-se por estar amplamente distribuído na América do Sul, tendo sido associado ao parasitismo de ovos da lagarta-enroladeira (Monteiro et al., 2004) e também da "grafolita" (Pinto et al., 2002).

Dessa forma, estudos que avaliem o potencial de inseticidas reguladores de crescimento e efeitos secundários são imprescindíveis no desenvol- vimento de programas de manejo integrado de insetos-praga nos agroecossistemas brasileiros, inclusive na cultura da macieira. Estes inseticidas, caracterizam-se por apresentarem baixa toxicidade a vertebrados e a muitos artrópodes benéficos por sua ação essencialmente de ingestão, o que lhes confere um alto grau de seletividade em relação a insetos e outros artrópodes que não ingerem a folhagem tratada. A utilização destes produtos é referida como componente viável em programas de manejo de pragas (Narayana \& Babu, 1992). Estudos anteriores têm demonstrado a seleletividade destes produtos a parasitóides de ovos (Bull \& Coleman, 1985, Zaki \& Gesraha, 1987, Cônsoli et al., 1998, Carvalho et al., 2002, Carvalho et al., 2003, Canete, 2005).

No entanto, segundo resultados já existentes verifica-se que a metodologia empregada pela International Organization for Biological and Integrated Control (IOBC) (Hassan et al., 2000), para testes de seletividade com reguladores de crescimento, não apresentam resultados satisfatórios, necessitando estudos para verificar alternativas mais eficazes para verificação do efeito destes produtos sobre os parasitóides de ovos do gênero Trichogramma.

Diante das atuais circunstâncias, foram realizados experimentos testando metodologias alternativas para verificar o efeito de inseticidas reguladores de crescimento na cultura da macieira sobre $T$. pretiosum, em comparação com a metodologia atualmente utilizada pela IOBC.

\section{MATERIAL E MÉTODOS}

Este trabalho foi realizado nos Laboratórios de Biologia de Insetos e de Pesticidas e Drogas do Departamento de Fitossanidade, Faculdade de Agronomia "Eliseu Maciel", Universidade Federal de Pelotas (UFPel), RS, na temperatura de $25 \pm 1^{\circ} \mathrm{C}$, UR $70 \pm 10 \%$ e fotofase de 14 horas.

Para a realização dos experimentos, utilizaram-se os seguintes inseticidas reguladores de 
crescimento, cujas concentrações indicadas entre parênteses expressam mL/100L água: lufenuron/ Match ${ }^{\circledR} \mathrm{CE}$ (300), methoxifenozide/Intrepid ${ }^{\circledR} 240$ SC (180), tebufenozide/Mimic 240 SC (90), flufenoxuron/Cascade 100 (100), e como inseticida padrão utilizou-se o inseticida fosforado fenthion (Lebaycid 500) (100).

\section{EFEITO DE INSETICIDAS REGULADORES DE CRESCIMENTO SOBRE TRICHOGRAMMA PRETIOSUM}

A medodologia de avaliação seguiu a preconizada pela IOBC/WPRS para testes de seletividade com parasitóides do gênero Trichogramma (Hassan et al., 2000). A aplicação dos inseticidas foi realizada com pulverizador manual de $580 \mathrm{~mL}$ da marca Guarany ${ }^{\circledR}$, proporcionando um depósito de calda entre 1,5 a 2,0 mg.cm ${ }^{-2}$, aferido mediante balança eletrônica de precisão.

Os parasitóides T. pretiosum utilizados nos experimentos foram oriundos de criação em laboratório, em câmaras climatizadas (temperatura $25 \pm 1^{\circ} \mathrm{C}$, UR $70 \pm 10 \%$ e fotofase 14 horas) utilizando-se ovos do hospedeiro alternativo Anagasta kuehniella (Zeller, 1879) (Lepidoptera: Pyralidae) inviabilizados em lâmpada germicida, criado conforme metodologia descrita por Parra (1997).

Os testes de toxicidade foram conduzidos expondo-se os adultos (estágio mais sensível) de $T$. pretiosum a resíduos secos dos pesticidas pulverizados sobre placas de vidro ( $2 \mathrm{~mm}$ de espessura e tamanho de $13 \mathrm{~cm}$ x $13 \mathrm{~cm}$ ), permanecendo estas, após a pulverização, à sombra por 3 horas para secagem da calda, formando uma película seca do produto-teste.

Tubos de emergência contendo adultos de T. pretiosum, com aproximadamente 24 horas de idade, foram conectados as gaiolas de exposição seis horas após a pulverização, permitindo a entrada dos insetos no interior da gaiola. Cada tubo de emergência foi constituído por uma ampola de vidro transparente de $120 \mathrm{~mm}$ de comprimento por $20 \mathrm{~mm}$ de diâmetro em uma das extremidades e 7 $\mathrm{mm}$ na outra, contendo no seu interior, um círculo de $1 \mathrm{~cm}$ de diâmetro com $250 \pm 50$ ovos previamente parasitados, aderido a uma tira de papel de $80 \mathrm{~mm}$ de comprimento por $15 \mathrm{~mm}$ de largura com três finos filetes de alimento $(3 \mathrm{~g}$ de gelatina, $100 \mathrm{~mL}$ de água e $200 \mathrm{~g}$ de mel). Após 24 horas da pulverização, círculos de $1 \mathrm{~cm}$ de diâmetro (450 \pm 50 ovos de A. kuehniella) foram ofertados no segundo dia (nove círculos), terceiro dia (seis círculos) e quinto dia (três círculos) após a pulverização, para serem parasitados, permitindo a avaliação do parasitismo e conseqüente efeito dos tratamentos.

A partir dos resultados do número de ovos parasitados e do número de fêmeas no interior da gaiola, obteve-se o número médio de ovos parasitados por fêmea de $T$. pretiosum para cada tratamento. Com base no parasitismo, os pesticidas-teste foram classificados em quatro categorias, conforme a IOBC em: 1) inócuo (<30\%) de redução de inimigos naturais; 2) levemente nocivo (30-79\%) de redução de inimigos naturais; 3) moderadamente nocivo (80-99\%) de redução de inimigos naturais; 4) nocivo (>99\%) de redução de inimigos naturais. Foram utilizadas quatro repetições para cada tratamento, sendo cada gaiola de exposição considerada uma unidade experimental.

\section{ENSAIO COM PULVERIZAÇÃO DE OVOS COM INSETICIDAS}

O experimento foi realizado adaptando a metodologia de Carvalho et al. (1994), sendo que os inseticidas foram aplicados com pulverizador manual de $580 \mathrm{~mL}$ da marca Guarany ${ }^{\circledR}$, proporcionando um depósito de calda entre 1,5 a 2,0 mg.cm-2 , aferido mediante balança eletrônica de precisão, nas cartelas contendo os ovos do hospedeiro (A. kuehniella) nas caldas químicas, sendo, em seguida, mantidos em condições ambientais por cerca de uma hora, para a eliminação do excesso de umidade de suas superfícies. Após a pulverização, as cartelas foram distribuídas individualmente em tubos de vidro (10 x $1 \mathrm{~cm})$, contendo uma fêmea de $T$. pretiosum. Estas fêmeas foram alimentadas com gotas de mel colocadas nas paredes dos tubos. Os tubos foram colocados em sala climatizada à temperatura de $25 \pm 2^{\circ} \mathrm{C}$, umidade relativa de $80 \pm 10 \%$ e fotofase de 14 horas, seguindo o delineamento estatístico inteiramente casualizado com 20 repetições, sendo cada repetição constituída por uma fêmea. Após o período de 24 horas de parasitismo, as cartelas foram transferidas para outros tubos, para avaliação da sobrevivência e longevidade da fêmea; número de ovos parasitados por fêmea (mediante a contagem de ovos do hospedeiro que tornaram-se escuros. O cório do ovo do hospedeiro parasitado torna-se escurecido em função da deposição de grânulos de urato que se inicia no estágio de pré-pupa do 
parasitóide (Cônsoli et al., 1999), viabilidade dos ovos parasitados (percentagem de emergência de adultos) e razão sexual dos descendentes (foi obtida através da contagem do número de fêmeas e machos, separados por caracteres morfológicos presentes nas antenas). Aos resultados obtidos aplicou-se a fórmula:

$\frac{\mathrm{rs}=\mathrm{n} .^{\mathrm{o}} \text { de fêmeas }}{\mathrm{n} .^{\mathrm{o}} \text { de fêmeas }+\mathrm{n} .^{\mathrm{o}} \text { de machos }}$

O número de parasitóides por ovo foi obtido dividindo-se a soma do número de fêmeas e número de machos pelo número de ovos parasitados, no período de 24 horas.

\section{ENSAIO COM INSETICIDAS MISTURADO EM SOLUÇÃO DE MEL}

Fêmeas de T. pretiosum em número de 20, recém emergidas foram individualizadas em tubos de vidro $(10$ x $1 \mathrm{~cm})$ e alimentadas com solução de mel contendo os inseticidas citados anteriormente, conforme metodologia adaptada de Canete (2005).

$\mathrm{O}$ mel foi trocado diariamente e a longevidade das fêmeas foi registrada. Juntamente com as fêmeas, círculos contendo ovos de A. kuehniella foram ofertados para cada fêmea, registrando-se o número de ovos parasitados e razão sexual dos descendentes. A comparação das médias dos parâmetros avaliados nos tratamentos foi realizada pelo teste de agrupamento de Scott e Knott a 5\% de probabilidade (Scott \& Knott, 1974).

\section{RESULTADOS E DISCUSSÃO}

Com relação à longevidade das fêmeas de T. pretiosum, na metodologia de pulverização de ovos, o inseticida fosforado Lebaycid, foi aquele que permitiu menor longevidade, sendo que os demais produtos igualaram-se ao tratamento testemunha (Tabela 1). Na metodologia utilizando-se mel adicionado aos com inseticidas reguladores de crescimento, Intrepid, Mimic, e o inseticida fosforado Lebaycid, os resultados obtidos igualaram-se os encontrados no tratamento testemunha, não levando a alterações na longevidade, enquanto os demais produtos (Match e Cascade) permitiram uma maior longevidade em relação à testemunha. Assim como demonstrado por Canete (2005), também neste estudo verificou-se que a ingestão de mel contendo inseticidas reguladores de crescimento não afetou a longevidade das fêmeas de T. pretiosum. Zaki \& Gesraha (1987) não constataram efeito adverso na longevidade de $T$. evanescens devido à ingestão da solução de mel contendo diflubenzuron, sendo esta a única referência sobre o efeito da ingestão de inseticidas por espécies de Trichogramma.

Comparando-se as metodologias testadas, os inseticidas Match, Intrepid, Mimic e testemunha apresentaram maior longevidade na metodologia na qual pulverizou-se os ovos, exceto para o inseticida Cascade, sendo que os demais produtos igualaramse estatisticamente (Tabela 1).

No parâmetro, ovos parasitados, todos os inseticidas reguladores de crescimento permitiram o mesmo valor nas duas metodologias testadas, com exceção do Lebaycid que apresentou número menor em relação à testemunha. Estes resultados concordam com Canete (2005), no qual fêmeas alimentadas com mel contendo reguladores de crescimento não apresentam diferenças estatísticas comparando-se ao tratamento testemunha. Os inseticidas Lebaycid e Intrepid foram similares entre as metodologias, enquanto que os inseticidas aplicados em pulverização resultaram em menor número de ovos parasitados, quando comparados com o experimento com mel adicionado a inseticida. Por outro lado, Zaki \& Gesraha (1987) constataram diminuição no número de ovos parasitados por T. evanescens alimentados com mel contendo diflubenzuron em relação à testemunha.

Com relação à razão sexual não ocorreu diferença estatística entre produtos, com exceção do Lebaycid, como também entre metodologias. Resultado semelhante encontraram Pratissoli et al. (2004), que não encontraram alteração na razão sexual dos descendentes quando os ovos do hospedeiro estavam em contato com inseticidas reguladores de crescimento. Carvalho et al. (1994), em teste com diversos reguladores de crescimento, também não observaram alteração no número de ovos parasitados e na razão sexual de T. pretiosum. À semelhança desses resultados, Suh et al. (2000) não observaram alteração na razão sexual de Trichogramma exiguum em relação à testemunha, quando ovos de Helicoverpa zea (Lep: Noctuidae) parasitados foram imersos nas caldas de methoxyfenozide e tebufenozide em suas concentrações comerciais. 
Referindo-se à taxa de emergência, o inseticida Lebaycid foi aquele que provocou a menor taxa de emergência seguido do inseticida Match, sendo que os demais igualaram-se à testemunha. Carvalho et al. (2002), também verificaram que o inseticida Match quando pulverizado em ovos de A. kuehniella reduziu significativamente a emergência de $T$. pretiosum. Resultados opostos encontrou Canete (2005), verificando que o número de adultos de $T$. atopovirilia emergidos destes ovos foi estatisticamente maior nos tratamentos com reguladores de crescimento em relação à testemunha. Suh et al. (2000) não observaram alteração na taxa de emergência de $T$. exiguum em relação à testemunha, quando ovos de H. zea parasitados foram imersos nas caldas de methoxyfenozide e tebufenozide.

$\mathrm{Na}$ metodologia contendo inseticida com mel, verificou-se que o inseticida Lebaycid, não apresentou emergência, enquanto que os demais produtos igualaram-se estatisticamente.

Quando se compararam as metodologias, verificou-se que o inseticida Intrepid em pulverização apresentou uma maior emergência, enquanto os demais inseticidas apresentaram resultados similares não diferindo estatisticamente (Tabela 1).

De acordo com as escalas propostas pela IOBC, pode-se observar na Tabela 2 que o inseticida Lebaycid utilizado como inseticida padrão

\section{Tabela 1}

Efeito de inseticidas reguladores de crescimento sobre a longevidade, número de ovos parasitados, razão sexual e taxa de emergência de Trichogramma pretiosum em duas metodologias alternativas. Temperatura $25 \pm 1^{\circ} \mathrm{C}$; UR: $70 \pm 10 \%$; Fotofase: 14 horas. Pelotas, RS, 2005.

\begin{tabular}{|l|l|l|l|l|l|l|l|}
\hline Parâmetros & & \multicolumn{1}{c|}{ Match } & \multicolumn{1}{c|}{ Intrepid } & \multicolumn{1}{c|}{ Mimic } & \multicolumn{1}{c|}{ Cascade } & \multicolumn{1}{c|}{ Lebaycid } & \multicolumn{1}{c|}{ Testemunha } \\
\hline \multirow{2}{*}{$\begin{array}{l}\text { Longevidade } \\
\end{array}$} & Pulv & $4,25 \pm 0,07 \mathrm{Aa}$ & $3,80 \pm 0,07 \mathrm{Aa}$ & $3,35 \pm 0,08 \mathrm{Aa}$ & $3,15 \pm 0,07 \mathrm{Aa}$ & $1,00 \pm 0,0 \mathrm{Ba}$ & $3,75 \pm 0,06 \mathrm{Aa}$ \\
\cline { 2 - 8 } & Mel & $2,70 \pm 0,06 \mathrm{Ab}$ & $0,70 \pm 0,07 \mathrm{Bb}$ & $0,50 \pm 0,08 \mathrm{Bb}$ & $3,25 \pm 0,08 \mathrm{Aa}$ & $1,05 \pm 0,02 \mathrm{Ba}$ & $1,30 \pm 0,09 \mathrm{Bb}$ \\
\hline $\begin{array}{l}\text { Ovos } \\
\text { Parasitados }\end{array}$ & Pulv & $15,40 \pm 0,17 \mathrm{Ab}$ & $23,60 \pm 0,16 \mathrm{Aa}$ & $18,35 \pm 0,18 \mathrm{Ab}$ & $16,90 \pm 0,19 \mathrm{Ab}$ & $0,35 \pm 0,05 \mathrm{Ba}$ & $18,50 \pm 0,17 \mathrm{Ab}$ \\
\cline { 2 - 8 } & Mel & $30,05 \pm 0,25 \mathrm{Aa}$ & $30,40 \pm 0,24 \mathrm{Aa}$ & $35,20 \pm 0,20 \mathrm{Aa}$ & $31,50 \pm 0,25 \mathrm{Aa}$ & $0,00 \pm 0,0 \mathrm{Ba}$ & $39,60 \pm 0,18 \mathrm{Aa}$ \\
\hline $\begin{array}{l}\text { Razão } \\
\text { Sexual }\end{array}$ & Pulv & $0,62 \pm 0,03 \mathrm{Aa}$ & $0,65 \pm 0,03 \mathrm{Aa}$ & $0,57 \pm 0,03 \mathrm{Aa}$ & $0,44 \pm 0,03 \mathrm{Aa}$ & $0,05 \pm 0,02 \mathrm{Ba}$ & $0,48 \pm 0,03 \mathrm{Aa}$ \\
\cline { 2 - 8 } & Mel & $0,43 \pm 0,03 \mathrm{Aa}$ & $0,39 \pm 0,03 \mathrm{Aa}$ & $0,40 \pm 0,03 \mathrm{Aa}$ & $0,61 \pm 0,03 \mathrm{Aa}$ & $0,00 \pm 0,0 \mathrm{Ba}$ & $1,20 \pm 0,02 \mathrm{Aa}$ \\
\hline $\begin{array}{l}\text { Taxa de } \\
\text { Emergência }\end{array}$ & Pulv & $0,35 \pm 0,04 \mathrm{Ba}$ & $0,70 \pm 0,03 \mathrm{Aa}$ & $0,72 \pm 0,04 \mathrm{Aa}$ & $0,52 \pm 0,03 \mathrm{Aa}$ & $0,07 \pm 0,02 \mathrm{Ca}$ & $0,65 \pm 0,03 \mathrm{Aa}$ \\
\cline { 2 - 8 } & Mel & $0,56 \pm 0,03 \mathrm{Aa}$ & $0,43 \pm 0,03 \mathrm{Ab}$ & $0,71 \pm 0,03 \mathrm{Aa}$ & $0,53 \pm 0,03 \mathrm{Aa}$ & $0,00 \pm 0,0 \mathrm{Ba}$ & $0,00 \pm 0,0 \mathrm{Bb}$ \\
\hline
\end{tabular}

2 Médias ( \pm EP) com a mesma letra maiúscula na linha e minúscula na coluna para cada parâmetro não diferem entre si pelo teste de Scott \& Knott à 5\% de probabilidade de erro.

Tabela 2

Toxicidade dos inseticidas reguladores de crescimento avaliados na capacidade de parasitismo de Trichogramma pretiosum em laboratório, através da metodologia padrão da IOBC. Temperatura $25 \pm 1^{\circ} \mathrm{C}$; UR: $70 \pm 10 \%$; Fotofase: 14 horas. Pelotas, RS, 2005

\begin{tabular}{|l|c|c|c|c|}
\hline \multicolumn{1}{|c|}{ Nome Comercial } & DC & Ovos parasitados por fêmea $^{\mathbf{2}}$ & RP $(\boldsymbol{\%})^{\mathbf{3}}$ & Classe de toxicidade $^{\mathbf{4}}$ \\
\hline Água Destilada & - & $32,40 \pm 2,23 \mathrm{a}$ & 0,00 & - \\
\hline Match $^{\circledR}$ & 300 & $4,73 \pm 2,27$ b & 85,40 & 3 \\
\hline Intrepid $^{\circledR}$ & 180 & $36,45 \pm 2,17 \mathrm{a}$ & 9,65 & 1 \\
\hline Mimic $^{\circledR}$ & 90 & $29,66 \pm 4,68$ a & 8,44 & 1 \\
\hline Cascade $^{\circledR}$ & 100 & $32,66 \pm 3,50 \mathrm{a}$ & 9,32 & 1 \\
\hline${\text { Lebaycid } 500^{\circledR}}^{\circledR}$ & 100 & $0,00 \pm 0,00$ b & 100,00 & 4 \\
\hline
\end{tabular}

1 DC = Dosagem da formulação comercial (g ou mL.100 L-1).

2 Médias acompanhadas pela mesma letra não diferem significativamente pelo teste de Tukey $(\mathrm{P}<0,05)$.

$3 \mathrm{RP}=$ Redução na capacidade de parasitismo comparado com a testemunha.

4 Classes da IOBC para teste de toxicidade sobre adultos: 1=inócuo (<30\%), 2=levemente nocivo (30-79\%), 3=moderadamente nocivo $(80-99 \%)$, 4=nocivo (>99\%). 
foi enquadrado na classe 4 (nocivo), seguido do inseticida Match classe 3 (moderadamente tóxico) porém, os demais foram classificados como classe 1 (inócuos).

Considerando-se que a metodologia empregada pela IOBC não apresenta resultados satisfatórios para testes de seletividade com produtos reguladores de crescimento, pelo fato desses produtos terem maior ação por ingestão e menor por contato as metodologias alternativas propostas neste trabalho, apresentam-se como uma forma de realização de testes de seletividade visto que os resultados destas metodologias foram semelhantes.

\section{CONCLUSÕES}

O inseticida fosforado Lebaycid utilizado com inseticida padrão apresenta maior toxidez em $T$.

\section{REFERÊNCIAS BIBLIOGRÁFICAS}

BULL, D.L. \& COLEMAN, R.J. 1985. Effects of pesticides Trichogramma spp. Southwest. Entomological Supplement 8: $156-168$.

CANETE, C.L, 2005. Seletividade de inseticidas a espécies de Trichogramma (Hymenoptera: Trichogrammatidae). Curitiba, 2005.117p. Tese (Doutorado em Ciências-Zoologia) - Universidade Federal do Paraná.

CARVALHO, G.A.; TIRONI, P.; RIGITANO, R.L.O. \& SALGADO, L. O. 1994. Seletividade de inseticidas reguladores de crescimento de insetos à Trichogramma pretiosum Riley (Hymenoptera: Trichogrammatidae). Anais da Sociedade Entomológica do Brasil 23: (3): 431-434.

CARVALHO, G. A.; REIS, P. R.; MORAES, J.C.; FUINI, L. C.; ROCHA, L.C.D.; GOUSSAIN, M. M. 2002. Efeito de produtos fitossanitários utilizados na cultura do tomateiro (Lycopersicon esculentum), a Trichogramma pretiosum Riley (Hymenoptera: Trichogrammatidae). Ciência e Agrotecnologia, 26: (6): 1160-1166.

CARVALHO, G.A.; PARRA, J.R.P. \& BAPTISTA, G.C. 2003. Efeito de produtos fitossanitários utilizados na cultura do tomateiro (Lycopersicon esculentum Mill.) a Trichogramma pretiosum Riley, 1879 (Hymenoptera: Trichogrammatidae) nas gerações $\mathrm{F}_{1} \mathrm{e}_{2}$ em ovos de Anagasta kuehniella (Zeller, 1879). Ciência e Agrotecnologia, 27: 295-304.

CÔNSOLI, F.L.; PARRA, J.R.P. \& HASSAN, S.A. 1998. Sideeffects of insecticides used in tomato fields on the egg parasitoid Trichogramma pretiosum Riley (Hym., Trichogrammatidae), a natural enemy of Tuta absoluta (Meyrick) (Lep., Gelechiidae). Journal Applied Entomology. 122: 43-47.

CÔNSOLI, F.L.; ROSSI, M.M. \& PARRA, J.R.P. 1999. Developmental time and characteristics of the immature stages of Trichogramma galloi and T. pretiosum (Hymenoptera, Trichogrammatidae). Revista Brasileira de Entomologia, 43: 271-275. pretiosum nas metodologias testadas, confirmando o resultado da IOBC, que classificou o produto como tóxico (> 99\% RP);

Em testes de seletividade com T. pretiosum recomenda-se utilizar as metodologias de pulverização de ovos e adição de inseticida ao mel como alternativas da metodologia padrão da IOBC por apresentarem resultados similares.

\section{AGRADECIMENTOS}

Apoio: bolsa de estudos da Coordenação de Aperfeiçoamento de Pessoal de Nível Superior (CAPES), Conselho Nacional de Desenvolvimento Científico e Tecnológico (CNPq). Bolsista CAPES: Morandi Filho, W.J., Bolsistas CNPq: GIOLO, F.P.; HÄRTER, W.R. Pesquisadores do CNPq: Grützmacher, A.D.

HASSAN, S. A., HALSALL, N., GRAY, A. P., KUEHNER, C., MOLL, M., BAKKER, F. M., ROEMBKE, J., YOUSEF, A., NASR, F., ABDELGADER, H. 2000. A laboratory method to evaluate the side effects of plant protection products on Trichogramma cacoeciae Marchal (Hym., Trichogrammatidae). In: CANDOLFI, M.P. et al. (Ed.). Guidelines to evaluate side-effects of plant protection products to non-target arthropods. [S.1.]: IOBC/ WPRS, p. 107-119.

KOVALESKI, A \& RIBEIRO, L.G. 2003. Manejo de pragas na produção integrada de maçã. In: PROTAS, J.F.da. S.; SANHUEZA, R.M.V. Produção integrada de frutas: o caso da maçã no Brasil. Bento Gonçalves :Embrapa Uva e Vinho, 2003. p.61-68.

MONTEIRO, L. B.; SOUZA, A.; BELLI, L.; QUERINO, R. B. \& ZUCCHI, R. A. 2004. Ocorrência de Trichogramma pretiosum Riley, 1879 (Hymenoptera: Trichogrammatidae) em ovos de Bonagota cranaodes (Meyrick, 1937) (Lepidoptera: Tortricidae) em macieira. Revista Brasileira de Fruticultura, 26: 171-172.

NARAYANA, M.L. \& BABU, T.R. 1992. Evaluation of five insect growth regulators on the egg parasitoid Trichogramma chilonis (Ishii) (Hym., Trichogrammatidae) and the hatchability of Corcyra cephalonica Staint (Lep., Galleriidae). Journal Applied Entomology. (113): 56-60.

PARRA, J.R.P. 1997. Técnicas de criação de Anagasta kueniella, hospedeiro alternativo para produção de Trichogramma. In: PARRA, J.R.P.; ZUCCHI, R.A. (Ed.). Trichogramma e o controle biológico aplicado. Piracicaba: FEALQ, 1997. Cap. 4, p.121-150.

JOHN D. PINTO; A. BERTHA KOOPMANSCHAP; GARY R. PLATNER \& RICHARD STOUTHAMER 2002. The North American Trichogramma (Hymenoptera: Trichogrammatidae) parasitizing certain Tortricidae (Lepidoptera) on apple and 
pear, with ITS2 DNA characterizations and description of a new species. Biological Control, 23: 134-142, 2002.

PRATISSOLI, D.; THULER, R.T. ; PEREIRA, F.F.; REIS, E.F. DOS \& FERREIRA, A.T. 2004. Ação transovariana de Lufenuron (50 G/L) sobre adultos de Spodoptera frugiperda (J. E. Smith) (Lepidoptera: Noctuidae) e seu efeito sobre o parasitóide de ovos Trichogramma pretiosum Riley (Hymenoptera: Trichogrammatidae). Ciência e Agrotecnologia, 28 (1): 9-14.

RIBEIRO, L.G. 1999. Principais pragas da macieira. In: Bonetti, J.I.S. da et al. Manual de identificação de doenças e pragas da macieira. Florianópolis : EPAGRI, 1999. Cap.3, p.97-149.

RIBEIRO, L.G. \& FLORES, E.H. 2002. Pulgão-verde: Aphis citricola Van der Goot (Homoptera: Aphididade). In:
EPAGRI. A cultura da macieira. Florianópolis : EPAGRI, 2002. Cap.15, p.519-521.

SCOTT, A.J. \& KNOTT, M.A. 1974. A cluster analysis method for grouping means in the analysis of variance. Biometrics, 30: 507-512.

SUH, C.P.C.; ORR, D.B. \& VAN DUYN, J.W. 2000. Effect of insecticides on Trichogramma exiguum (Hymenoptera: Trichogrammatidae) preimaginal development and adult survival. Journal of Economic Entomology, 93 (3): 577-583.

ZAKI, F.N. \& GESRAHA, H.A. 1987. Evaluation of zertel and diflubenzuron on biological aspects of the egg parasitoid, Trichogramma evanescens Westw. and the aphid lion Chrysoperla carnea Steph. Journal Applied Entomology, 104: 63-69. 